\title{
Der sozialwissenschaftliche Film
}

\section{Möglichkeiten und Grenzen ${ }^{1}$}

\section{Katharina Miko-Schefzig}

Journal für Psychologie, 28(1), 134-155

https://doi.org/10.30820/0942-2285-2020-1-134

CC BY-NC-ND 3.0 DE

www.journal-fuer-psychologie.de

\section{Zusammenfassung}

Seit dem sogenannten Visual Turn wird auch dem Einsatz von Film in den Sozialwissenschaften mehr Aufmerksamkeit geschenkt. Dabei ist die Nutzung zweigeteilt: einerseits als Erhebungs- und Auswertungsmethode, andererseits als wissenschaftlicher Output. In diesem Artikel werden die Herausforderungen und Potenziale der Produktion sozialwissenschaftlicher Filme diskutiert. Es wird sowohl auf methodologische und theoretische Fragen wie auf Fragen der konkreten Umsetzung eingegangen. Zentrale Schritte im Forschungsprozess werden in ihrer Bedeutung für die Filmarbeit diskutiert. Umgekehrt werden deren spezifische Stilmittel, etwa der Schnitt, in ihrer Bedeutung für die empirische Arbeit dargestellt. Schlussendlich werden die Folgen eines vermehrten Einsatzes von Film für das Wissenschaftssystem, etwa für die Publikationsmöglichkeiten, beleuchtet.

Schlüsselworte: Sozialwissenschaftlicher Film, performative Sozialwissenschaft, Visualität, visuelle Sozialwissenschaft, Repräsentation

\section{Summary}

The social science film. Chances and challenges

Since the so-called visual turn in the social sciences, more attention has been paid to the use of film. The general debate focuses mainly on two aspects: on the one hand, film as a method of data collection and analysis, on the other hand, film as a scientific output. This article discusses the challenges and potentials of producing social science films. Methodological and theoretical questions as well as practical questions of the actual film work are addressed. Central steps in the research process are discussed in their significance for film work. Conversely, central steps in the film process, such as editing, are presented in their significance for the research process. Finally, the consequences of an increased use of film in the social sciences will be examined in terms of their consequences for the scientific system, such as publication strategies.

Keywords: social science film, performative social science, visuality, visual studies, representation 


\section{Einleitung}

Der sozialwissenschaftliche Film hat in der wissenschaftlichen Community in den letzten Jahren Anerkennung gewonnen - etwa im Hinblick auf die Produktion, die Effekte, die er auf eine wortzentrierte Wissenschaftspraxis hat, oder das Potenzial, das man allgemein in der performativen Sozialwissenschaft sieht (Banks und Zeitlyn 2015; Christianson 2016; Denzin 2001; Glass 2008; Glisovic, Berkeley und Batty 2016; Guiney Yallop, Lopez de Vallejo und Wright 2008; Holm 2008; Jarzabkowski, Bednarek und Cabantous 2015; Kurt 2010; Linstead 2018a; Mey 2018; Miko 2013; Pink 2007; Rose 2014; Schändlinger 1998; Slutskaya, Game und Simpson 2018; Vannini 2015; Wood und Brown 2011; Wood, Salovaara und Marti 2018). Tatsächlich gibt es einige Filme, die ganz originär Forschungsergebnisse darstellen - und dies zum Teil mit großem Erfolg sowohl in den Sozialwissenschaften als auch nach den Kriterien des konventionellen Dokumentarfilms, etwa: Black Snow (Linstead 2018b), Auf den Spuren von Martha Muchow (Mey und Wallbrecht 2016), Warme Gefüble - vier Liebesgeschichten aus Österreich (Miko und Frick 2012), Kinder als Grenzgänger. Übergangspraktiken im Betreuungsalltag (Mohn 2016), Leadership in Spaces and Places (Salovaara 2014), Life off Grid (Taggart 2016) und Lines of Flight (Wood und Brown 2014).

Diese Filme könnten als Dokumentarfilme bezeichnet werden - ein Begriff, der in den Sozialwissenschaften breit verwendet wird (z. B. Slutskaya, Game und Simpson 2018). Es gibt jedoch wesentliche Unterschiede zwischen sozialwissenschaftlich produzierten Filmen und Dokumentarfilmen im Fernseh- und Kinobereich (zu den Gemeinsamkeiten zwischen »sozialwissenschaftlicher und filmdokumentarischer Erfahrungsbildung « siehe Schändlinger 1998, 11). In erster Linie wird ein sozialwissenschaftlicher Film im akademischen Kontext produziert und dient vor allem dazu, der sozialwissenschaftlichen Community Forschungsergebnisse zu vermitteln. Wood, Salovaara und Marti $(2018,825 f$.) verwenden etwa den allgemeinen Begriff $»$ film $\ll$, kontextualisieren ihn aber als »academic film « oder »film-based research «. Mit solchen Filmen wird, wie etwa auch mit Beiträgen in Zeitschriften, versucht, Wissen zu erweitern, vorhandene Theorien infrage zu stellen oder sozialwissenschaftliche Argumente zu liefern. Sozialwissenschaftliche Filme erfordern methodisches Vorgehen und eine Auseinandersetzung mit sozialwissenschaftlichen Theorien (Kaczmarek 2008; Kurt 2010; Miko und Sardadvar 2010). Ganz oder teilweise von Wissenschaftler/inne/n produziert, »dokumentieren « solche Filme nicht, sondern versuchen Aspekte unserer sozialen Welt neu zu theoretisieren. Zu den Subgenres des sozialwissenschaftlichen Films gehören etwa der ethnografische Film - ein Begriff, der traditionell in der Kultur- und Sozialanthropologie verwendet wird (Heinze und Weber 2017; Miko und Sardadvar 2008; Vannini 2015) - oder auch der soziologische Film (Kaczmarek 2008; Miko 2013). Ob- 
schon beide Begriffe plausibel sind, bevorzuge ich im Folgenden als ein umfassenderes, nichtdisziplinäres Label den allgemeineren Begriff sozialwissenschaftlicher Film (MikoSchefzig, Learmonth und McMurray 2020/in Begutachtung).

In diesem Artikel möchte ich die verschiedenen Aspekte des sozialwissenschaftlichen Films diskutieren und auch meine eigenen Wurzeln der Beschäftigung damit beleuchten, etwa eine seit 2007 regelmäßig durchgeführte Lehrveranstaltung am Institut für Soziologie der Universität Wien, in der bis jetzt um die 30 Filme produziert wurden. Im Sommersemester 2007 fanden sich meine Kollegin Karin Sardadvar und ich im Rahmen unserer Lehrtätigkeit zu einem Experiment zusammen: Wir wollten ein Seminar ausrichten, das ethnografische Forschung mit der Verwendung von Videomaterial verbinden sollte. In dieser Lehrveranstaltung waren Filmproduktion und die Reflexion darüber Teil der Methodenlehre. Konkret hatten die Studierenden im Seminar die Aufgabe, ein kleines ethnografisches Forschungsprojekt durchzuführen und dabei - in welcher Weise auch immer - mit Film zu arbeiten. Eine Möglichkeit bestand darin, den Endbericht teilweise durch einen Film zu ersetzen, also nicht nur gefilmtes Datenmaterial zu verwenden, sondern die Ergebnisse auch in filmischer Form zu verarbeiten und darzustellen. Es gab keine Vorgaben, die Studierenden waren aufgefordert, selbst Ideen und Möglichkeiten zu entwickeln. Einer der Gründe für diese Offenheit war, dass keine verbindlichen Richtlinien vorliegen, wie ein sozialwissenschaftliches filmisches Dokument herzustellen ist oder aussehen sollte. Wir konnten zwar Beispiele diskutieren, doch eine klare Antwort darauf, wie »der « sozialwissenschaftliche Film zu sein hat, ließ sich erwartungsgemäß nicht finden. Was vielmehr zutage trat, waren Varianten und Optionen der filmischen Darstellung, Schwierigkeiten und Potenziale, offene Fragen und mögliche Antworten.

\section{Vom ethnografischen zum sozialwissenschaftlichen Film}

Der sozialwissenschaftliche Film kann theoretisch unterschiedlich ein- und zugeordnet werden: als Teil der qualitativen Methodenlehre, als Teil des Visual Turns und somit als eine akademische Reaktion auf eine zunehmend visuelle Vergesellschaftung oder auch als Teil der performativen Sozialwissenschaft. Alle drei Debatten sind nur idealtypisch voneinander getrennt und bedingen einander bis zu einem gewissen Grad. Diese virtuelle Teilung spiegelt sich auch in diesem Artikel wider, wenngleich ich einen Fokus auf methodologische und methodische Fragestellungen lege. Eine Kritik der performativen Sozialwissenschaft an der dominanten Sprachorientierung im akademischen Umfeld ist, dass » sich im Wissenschaftssystem eine eigene Sprache durchgesetzt hat, die einem innerwissenschaftlichen Diskurs verpflichtet, [...] aber für interessierte Lai/innen bzw. Angehörige anderer Fachdisziplinen nicht zugänglich ist « (Mey 2018, 4). Diese Kritik 
ist nicht zu unterschätzen und führt letztendlich zum Kern methodischer beziehungsweise epistemischer Fragen oder wie es Figgis $(2007,3)$ in Hinblick auf die Verwendung von Kameras ausdrückt: »Suddenly you realise this is a whole different way of looking at things. «

\subsection{Abbildung versus Inszenierung: Von der vermeintlichen Authentizität des Bildes}

Sozialwissenschaftliche (vor allem ethnografische) Forschung und die Arbeit mit Film sind in ihrer Geschichte verbunden. Die Verwendung von Film zur Aufnahme von Daten findet sich schon in den 1930er Jahren bei den Ethnolog/inn/en Margaret Mead und Gregory Bateson (1942) in ihrer berühmt gewordenen Studie zum »balinesischen Charakter «, wo buchstäblich meterweise Fotografien und Filmmaterial angesammelt wurden (vgl. u. a. Wolff 1995). Diese Studie gab zumindest der Kultur- und Sozialanthropologie den Impuls, sich ab den 1950er Jahren verstärkt mit der Verwendung von audiovisuellem Material und der Produktion von Dokumentarfilmen zu beschäftigen (Denzin 2000, 418).

Die Ursprünge des ethnografischen Films gehen auf Filmemacher wie Robert J. Flaherty (Nanook, 1922) sowie den Anthropologen Jean Rouch und den Soziologen Edgar Morin (Chronique d'un été, 1961) zurück. Die Filme der beiden lösten Debatten über den sozialwissenschaftlichen Film aus, die für die Nutzung des Films als Form der wissenschaftlichen Kommunikation bis heute relevant sind, etwa in Fragen wie: Wie viel ist geschnitten? Ist dies wirklich das Leben der Beobachteten? Welchen wissenschaftlichen Mehrwert hat das Erstellen eines Films?

Ende des 19. Jahrhunderts entstanden bereits Filme mit ethnografischen und ethnologischen Inhalten, etwa Félix Louis Regnaults Aufnahmen von handwerklichen Tätigkeiten der afrikanischen Wolof (vgl. Pfeifer 2006). Die Anfänge des ethnografischen Films sind somit eng mit den Anfängen der Kinematografie selbst verbunden. Als besonderes Beispiel sei hier auf die Arbeiten der Gebrüder Lumière hingewiesen: Ihr erster Film zeigte Arbeiter/innen beim Verlassen der Lumière-Werke, einer Fabrik, in der fotografische Ausstattung hergestellt wurde. Für einen weiteren filmten sie die Ankunft eines Zuges. Bei den ersten Vorführungen verließen die Besucher/innen erschrocken ihre Plätze, weil sie dachten, dass die Menge der Arbeiter/innen beziehungsweise die Eisenbahn tatsächlich auf sie zugelaufen beziehungsweise zugerast käme.

$\mathrm{Zu}$ jener Zeit war Film also noch nicht derart selbstverständlich in die Nähe der Fiktion getreten, wie er es heute ist. Schon früh in der Filmgeschichte wurde der Unterschied zwischen Realität und Ästhetisierung, zwischen Dokumentation und 
Wirklichkeitsveränderung diskutiert. Was die Aufgabe des Films ist, das ist eine Diskussion, die seit dem Anfang des letzten Jahrhunderts die Gemüter bewegt (vgl. Monaco 1997). »Dies [die Filme der Brüder Lumière] waren einfache, doch eindrucksvolle Proto-Filme. Sie erzählten keine Geschichte, sondern sie gaben lediglich einen Ort, einen Zeitpunkt und eine Atmosphäre so wirkungsvoll wieder, dass das Publikum eifrig dafür zahlte, dieses Phänomen zu besichtigen « (Monaco 1997, 283). Der Filmemacher Georges Meliès, ein Zeitgenosse der Gebrüder Lumière, stand deren Arbeiten kritisch gegenüber und vertrat die Ansicht, dass Film die Realität verändern und nicht bloß darstellen sollte.

Seit der Krise der Repräsentation in den Sozialwissenschaften (Berg und Fuchs 1993) - also dem kritischen Hinterfragen des Verhältnisses zwischen Forscher/inne/n und beforschtem Feld sowie der Debatte um die Konstruktion des Forschungsfeldes durch den Forschungsfokus und die darauf aufbauende Repräsentation der Ergebnisse (siehe etwa Mohn 2002) - ist die Idee des simplen Abbildens von sozialer Realität durch Film in die Kritik geraten. Folgerichtig ist auch die Vorstellung, dass sozialwissenschaftlicher Film das Soziale authentisch zeigen könne (etwa Heider 2006), nicht mehr aktuell. Heider ging noch davon aus, dass die Angemessenheit eines Films für die Wissenschaft mehr oder weniger messbar wäre:

$\gg$ Etwas tautologisch ersetzt er > ethnographisch $<$ durch $>$ Anteil an Ethnographizität $<$ und löst dann den Begriff Ethnographizität in verschiedene Attribute auf, die er gemäß ihrer Wertigkeit in ein Polaritätsprofil überträgt, an dessen Kurve dann die Qualität (gleich Ethnographizität) des jeweiligen Films ablesbar ist « (Hohenberger 1988, 143).

Eines der Merkmale war zum Beispiel »ganze Handlungen «: Eine niedrige Ethnografizität wurde erreicht, wenn nur Ausschnitte einer Handlung gezeigt wurden, während die Darstellung einer Handlung von Anfang bis Ende eine hohe Ethnografizität bedeutete. Wenngleich Heiders Ansatz als überholt gilt, ist sein Anliegen - ein Kriterium zu finden, das die Anschlussfähigkeit an das wissenschaftliche System und somit eigentlich die Wissenschaftlichkeit des Films überzeugend belegen kann - nach wie vor relevant.

Ich bin bei der Produktion unseres sozialwissenschaftlichen Films (Miko und Frick 2012) von Anfang an einen anderen Weg gegangen. Da ich auch in einen wissenschaftlichen Artikel keine » ungeschnittenen « Beobachtungsprotokolle integriere beziehungsweise - pointierter formuliert - keine Beobachtungsprotokolle als Artikel » verkaufe «, ist für mich die Anwendung filmsprachlicher Mittel, wie etwa des Schnitts, durch Sozialwissenschaftler/innen unabdingbar. Sozialwissenschaftliche Filme wie unsere Arbeit Warme Gefüble - vier Liebesgeschichten aus Österreich können die Welt um uns herum zeigen und auf wissenschaftlicher Basis rekonstruieren. Kurt (2010) greift 
hier etwa auf Max Webers Konzept des Idealtypus zurück, das seiner Ansicht nach filmisch genutzt werden kann, um Verdichtungen zu schaffen:

»Für den filmischen Idealtypus heißt dies, dass er etwas aufzeigt, was sich so gar nicht ereignet hat: Hier wird eine Szene auf einen Bruchteil der Originalzeit zusammengekürzt, dort wird Späteres dem Früheren vorangestellt [...]. Entscheidend für die Konstruktion eines filmischen Idealtypus ist nicht, dass er das ursprüngliche Geschehen in Echtzeit und im Originalsynchronton wiedergibt, sondern dass er der Interpretationsfigur des Sozialwissenschaftlers Gestalt verleiht. Zu diesem Zweck scheint mir die Arbeit mit filmästhetischen Mitteln legitim zu sein « (Kurt 2010, 200).

In diesem Zusammenhang ist auch Streecks (2017) Analogie zwischen Schauen versus Sehen (looking vs. seeing) und Hinzeigen versus Herzeigen (pointing vs. showing: die deutsche Übersetzung bleibt hinter dem englischen Original zurück) mit der Kamera hilfreich. Ich möchte dies am Beispiel von Friedemann, einem Protagonisten aus unserem Film, veranschaulichen. In Warme Gefüble geht es um die Lebensgeschichten älterer lesbisch beziehungsweise schwul lebender Menschen. Die Basis des Films waren qualitative Interviews mit rund 30 Personen. Im Jahr 2010 wurde ich (gemeinsam mit der Historikerin Ines Rieder) gebeten, anlässlich einer großen Ausstellung in Wien mit dem Titel »100 Jahre Schwule und Lesben in Wien « Found Footage (also Archivfilme) auszuwählen und zu schneiden. Durch die Bearbeitung des Materials aus verschiedenen Jahrzehnten wurde mir bewusst, dass es lange vor der sexuellen Revolution der 1960er Jahre eine Art »hidden gay movement « gegeben hatte. Man könnte dieses Phänomen wahrscheinlich auch »in-the-closet-movement « nennen, was natürlich auch bedeutete, dass es eine unerzählte Geschichte geblieben war. Ines Rieder und ich begannen mit einem konventionellen Theoretical Sampling (Strauss und Corbin 1996 [1990]), indem wir die Hauptkriterien auswählten, die wir untersuchen wollten (z. B. Alter, Ausbildung). Alle Interviews wurden transkribiert und thematisch auf die wichtigsten Themen, Konzepte und Anliegen hin analysiert. Nachdem wir eine theoretische Sättigung erreicht hatten (Glaser und Strauss 1967), konnten wir für die 1950er Jahre Idealtypen (im Weber'schen Sinne) von schwulen und lesbischen Biografien konstruieren. Das Geschlecht war eines der zentralen Unterscheidungsmerkmale dieser Idealtypen, denn für Frauen war eine Lebensentscheidung gegen die Ehe zu dieser Zeit fast unmöglich oder hätte zumindest enorme wirtschaftliche Konsequenzen gehabt. Herta, eine Protagonistin im Film, sagte uns, dass sie sich eine Scheidung schlichtweg nicht hätte leisten können. Eine andere Hauptkategorie war der Bildungshintergrund beziehungsweise der berufliche Kontext: Der Protagonist Rudy etwa, Teil der Medienelite des Landes, lebte seine Homosexualität problemlos - solange er nicht darüber sprach. Im Gegensatz dazu landete Friedemann, ein Teppichweber, im Gefängnis. 
Ein Element des Filmes war das durch die Kamera begleitete Zurückgehen der Protagonist/inn/en zu einem wichtigen Lebensereignis, das für das (Er-)Leben ihrer sexuellen Orientierung einschneidend war. Friedemann wählte das Gefängnis. Er war als einer der letzten homosexuellen Männer in Österreich auf Basis des Totalverbots von Homosexualität $\mathrm{zu}$ - so wurde das damals genannt - »schwerem Kerker « verurteilt worden, bevor das Gesetz 1971 aufgehoben wurde. Während der Dreharbeiten forderte Friedemann uns auf, die Kamera zuerst auf den Zaun um das Gefängnis und später auf die umliegenden Felder zu richten (looking). Wir sahen zuerst nicht (seeing), was er meinte, aber dann sagte er: »Wenn es nicht so frivol klingen würde, würde ich sagen, schön ist es da. $\ll^{2}$ Wir verwendeten die Kamera als methodisches Werkzeug, um sowohl in das Gefängnis als auch auf die Felder dahinter zu schauen (looking), während Friedemann uns einlud, die Welt aus seiner Perspektive zu verschiedenen Zeiten und an verschiedenen Orten zu sehen (seeing). Die Zuschauenden können sehen (im wahrsten Sinne des Wortes), dass Friedemann eine neue (Kamera-)Perspektive auf ein Lebensereignis wirft. Während er auf die Felder zeigt, schwenkt die Kamera in dieselbe Richtung (pointing). Friedemann lädt uns ein, seine Geschichte zu sehen. Er zeigt uns im und durch den Film (showing), wie es war, zu einer anderen Zeit schwul zu sein, indem er die Szene aus dem Hier und Jetzt betrachtet. Im Kontext der Frage, welches wissenschaftliche Wissen mit sozialwissenschaftlichem Film erzeugt wird, ist die Konkretisierung des Begriffs »visuelles Wissen «, sowohl für die Aufnahme der Bilder als auch für Entscheidungen im Schnitt, relevant. Es macht beispielsweise einen Unterschied, ob Forscher/innen selbst die Kamera bedienen oder diese an die beforschte Gruppe weitergeben: »Geben Forscher ihre Kameras nicht aus der Hand, dann müssen sie aufpassen, dass sie mit ihren kulturell geprägten Seh- und Verstehensgewohnheiten nicht systematisch übersehen, was aus Sicht der Gefilmten Relevanz besitzt « (Kurt 2010, 197).

Sozialwissenschaftliche Filme sind besonders wertvoll, wenn Wissen und Ergebnisse gezeigt werden sollen, die außerhalb der gesprochenen und geschriebenen Sprache liegen (Emotionen, Körperwissen u. Ä.). Um »elusive knowledges « (»flüchtiges Wissen «; Toraldo et al. 2018) kommunizieren zu können, benötigen wir Methoden, die explizite, implizite, ästhetische und verkörperte Aspekte des sozialen Lebens vermitteln können. Dementsprechend haben sich Hassard et al. $(2018,1411)$ für » a more nuanced sense of affect and embodiment in video-based research « ausgesprochen. Die Dreharbeiten mit Friedemann haben uns dieser sinnlichen Erfahrung nähergebracht. Wenn wir nur ein Interview mit ihm geführt hätten, ohne mit einer Kamera ins Gefängnis zu gehen, wären uns nur Friedemanns Worte geblieben. Natürlich hätte die Rückkehr ohne Kamera in einer ethnografischen Studie auch ein methodisches Instrument sein können. Mit Film konnten wir jedoch den darstellenden, performativen Aspekt (Goffman 1974) jeder sozialen Situation nutzen und ihn mit der Kamera intensivieren, da Protagonist/inn/en dazu neigen, mit der Kamera zu interagieren, um ein bestimmtes 
Selbst zu präsentieren. Hassard et al. $(2018,1411)$ sprechen in diesem Zusammenhang (und auch in Bezugnahme auf Goffman) vom »dramaturgical body«, der über das Zusammenspiel mit der Kamera adressiert werden kann. Sarah Pink (2007) hat dies folgendermaßen zusammengefasst:

»The ways ethnographers intend to represent their research inevitably inform how they approach their projects, the technologies used, their relationships with informants and the experiences and knowledge they produce. These relationships, technologies and experiences might also be reflected in their representations. When video plays a key role in the research it seems appropriate to incorporate video in their representation « (Pink 2007, 172).

Im Folgenden möchte ich nun auf die Unterscheidung zwischen Video als Datenmaterial und Film als Alternative zum Endbericht eingehen. In diesem Zusammenhang komme ich auch auf ethische Aspekte der Forschungsarbeit mit Video sowie auf Ähnlichkeiten und Unterschiede zwischen Dokumentarfilm und sozialwissenschaftlichem Film zu sprechen. Abschließend diskutiere ich Fragen der Implementierung von Film in die Sozialwissenschaften, etwa die Aufnahme in Ausbildungscurricula.

\subsection{Film als Datenmaterial}

Im Rahmen qualitativer Forschung kann Videomaterial auf mehrere Arten verwendet werden, zum Beispiel als Datenmaterial: Hier dient die Kamera gewissermaßen als ein » zusätzliches Auge «, als eine Alternative zur schriftlichen Protokollierung von Beobachtungen oder Interviews. Wenn man im Feld per Video protokolliert, dann erfordert das Sensibilität hinsichtlich der Wirkung der Kamera auf die Menschen im Feld. Es gilt zu reflektieren, ob man mit dem »Fremdkörper « Kamera tatsächlich teilnehmend beobachten kann oder vielmehr die eigene Außenseiter/innenposition ständig zur Schau trägt. Außerdem bietet die Verwendung von Video die Chance, ein visuelles Protokoll der Beobachtungen im Feld zu erstellen, indem man die Situation nicht nur einmal, sondern immer wieder aufs Neue beobachten kann - und vielleicht beim vierten Ansehen Aspekte entdeckt, die bei einem einmaligen Beobachten der Aufmerksamkeit entschlüpft wären. Wir wissen, dass jeder der Sinne nicht unbedingt ein Mehr, sondern ein Anders der sozialen Fokussierung mit sich bringt. Mit Riach und Warren (2015; in einer Studie über die Sinneslosigkeit in den Sozialwissenschaften am Beispiel von Geruch bzw. Riechen) kann man vielleicht sagen: »We accept that to write about corporeal porosity is to ultimately limit it to a textual reduction that only offers a glimpse of the embodied processes at play« (ebd., 806). 
Für die Hinwendung zu Film als Datenmaterial kommt Forscher/inne/n die veränderte Wahrnehmung des Filmens entgegen. War noch vor 20 Jahren eine Person mit Kamera auf der Straße ein/e auffallende/r Akteur/in, so hat die Entwicklung der DVKameras vor rund 15 Jahren und deren zunehmende Verkleinerung das Straßenbild mit filmenden Menschen gefüllt. Seit dem Aufkommen von Smartphones mit eingebauten Kameras stellt Filmen im öffentlichen Raum eine Alltagspraxis dar. Bei der Verwendung von Kameras im Forschungsfeld ist auch auf ethische und rechtliche Aspekte hinzuweisen. Auf der rechtlichen Ebene ist das Filmen hinsichtlich des Urheberrechts zu überprüfen (im Besonderen $\gg$ das Recht am eigenen Bild $\ll^{3}$ ).

Auf der ethischen Ebene ist die Antwort einfacher und schwieriger zugleich. Einfacher, weil rechtlich klar geregelt ist, dass jede Person, die gezeigt oder gefilmt wird, ihre Zustimmung geben muss. Schwieriger, weil die Grenze des ethisch Vertretbaren subjektiv und fließend ist und jeweils im Kontext des konkreten Forschungsprojektes abgesteckt werden muss. ${ }^{4}$ Ein Beispiel: Ist das Filmen eines sterbenden Menschen im Spital »objektiv « ethisch unangebracht? Ist das Problem bereits gelöst, wenn die Person ihr Einverständnis gibt? Oder gehört zur ethischen Verantwortung auch das Miteinbeziehen einer möglichen Überforderung der Rezipient/inn/en? Derlei Fragen können an dieser Stelle nicht generell beantwortet werden, sollten aber für jede Forschungsarbeit im Hintergrund mitlaufen.

Zur Verwendung von Film im Rahmen der Datensammlung ist abschließend anzumerken, dass es sich bereits bei der Aufzeichnung um eine Deutung von Wirklichkeit handelt: »Filme oder Fotografien liefern ein Bild oder eine Ansammlung von Bildern als Deutungen von Wirklichkeit. Die Wirklichkeit oder der eingefangene Wirklichkeitsausschnitt kann niemals reproduziert werden, da das Gezeigte nur einmal geschehen kann $\ll($ Denzin 2000, 423).

\subsection{Film als Medium der Ergebnisdarstellung: Der sozialwissenschaftliche Film}

Eine andere Möglichkeit, in der sozialwissenschaftlichen Forschung mit Video zu arbeiten, ist der sozialwissenschaftliche Film als eine Form der Ergebnisdarstellung und Alternative (bzw. Ergänzung) zum schriftlichen Endbericht. Wenn man über den »sozialwissenschaftlichen Film « spricht, dann verbindet man dabei zwei gewichtige Felder - Wissenschaft und Kunst - und die damit verbundenen Ansprüche. Diese gilt es in unserem Kontext auseinanderzuhalten: Auf der einen Seite steht die Produktion von Film im akademischen Umfeld, auf der anderen Seite die Filmkunst.

Zwischen Dokumentarfilmen und dem sozialwissenschaftlichen Film gibt es Naheverhältnisse, aber auch klare Unterschiede. Allerdings haben sich die Produktionsweisen 
angeglichen. Während vor zwei Jahrzehnten die Produktion von professionellem Film und die Produktion von Laienvideos von einem technischen Standpunkt aus gesehen weit voneinander entfernt waren, sind heute häufig dieselben Codecs $^{5}$ (H.264 etc.) vorzufinden. Der für einen Oscar nominierte Film Darwin's Nightmare von Hubert Sauper (2005) etwa begann als Reisevideo des Regisseurs. Dies führt uns zu einem zweiten wesentlichen Punkt: Der/Die sozialwissenschaftlich Filmschaffende stellt in der Regel die Kamera auf und versucht zunächst einmal ohne Drehbuch, die Situation zu erfassen. Die Entstehung von Dokumentarfilmen läuft oft ähnlich ab. Man befindet sich im Feld, kennt es nicht und muss erst langsam erfahren, welches Material typisch ist.

Dennoch muss der sozialwissenschaftliche Film vom Dokumentarfilm ebenso wie von der Datenprotokollierung per Video abgegrenzt werden. Es geht hier um eine prinzipielle Unterscheidung, die sich mehr auf das Ziel bezieht als auf das Tun der Akteurinnen und Akteure. Das Ziel der Dokumentarfilmer/innen bleibt die filmische Darstellung, die filmische Ästhetisierung eines sozialen Phänomens. Ein/e Dokumentarfilmer/in verletzt kein Gütekriterium seiner/ihrer Profession, wenn er/sie sich für eine subjektive Positionierung entscheidet, wie beispielsweise Michael Moore, der sich vor die Kamera stellt und sehr persönliche Ansichten direkt an das Publikum richtet. Michael Moore wird vielleicht dafür kritisiert, nur eine Seite eines Phänomens darzustellen, ist aber noch immer Filmschaffender.

Wird in der Soziologie Film als Ersatz für einen Endbericht verwendet, darf der/die filmende Forscher/in nicht außerhalb der soziologischen Gütekriterien agieren. Denn bei einem wissenschaftlichen filmischen Endbericht geht es nicht um subjektive Darstellungen, sondern um die Darstellung von »filmischen Idealtypen « (Kurt 2010), die in einem klassisch durchgeführten Analyseprozess mittels soziologischer Methoden herausdestilliert wurden. Wo der Dokumentarfilm für den Fernseh- und Kinobereich auf das Prinzip Meinung und Gegenmeinung zurückgreift (also: Sagt die interviewte Person X etwas, muss die Gegenmeinung durch Person Y dargestellt werden), wählt die Wissenschaft eher die Typisierung. Durch das Hineinschneiden der Originalaussagen (analog den Zitaten im schriftlichen Text) ist die visuell dichte Beschreibung wesentlich schwieriger darzustellen als im schriftlichen Pendant (Miko-Schefzig, Learmonth und McMurray 2020/in Begutachtung). Erst nachdem man klassische soziologische Analysemethoden, beispielsweise die Feinstrukturanalyse nach Froschauer und Lueger (2003), angewandt hat, wird das Ergebnis filmisch umgewandelt. Das kann auch bedeuten, dass man zuerst filmt, um eine Bildanalyse durchführen zu können, und erst nach der Ergebnisdarstellung noch einmal ins Feld geht, um Typisches für die Filmproduktion zu filmen. Es gibt in der Bildbeschaffung keine festen Regeln. Dagegen sind die Regeln für »gutes « Forschen sehr klar ausformuliert. Beim sozialwissenschaftlichen Filmen gilt es diese Regeln einzuhalten, wie in jedem anderen Forschungsprojekt auch. 
Zusammengefasst bedeutet dies: Prinzipiell ist es das große Ziel des sozialwissenschaftlichen Films, eine andere Form des Endberichts, jenseits der sprachlichen Möglichkeiten, zu produzieren. Datenerhebung und -analyse finden dabei in derselben Weise statt wie bei einem Forschungsprojekt mit schriftlichem Abschlussbericht. Unterschieden sind die beiden Vorgangsweisen nur durch den Schnitt und andere filmische Gestaltungsmittel. Dazwischen aber liegt klassisches sozialwissenschaftliches Handwerk: das der Analyse.

\section{Vorstellungskraft und Dissemination: Wozu eine Alternative zum schriftlichen Endbericht?}

Ein Film als Alternative zum Endbericht in Textform eignet sich sicherlich nur für bestimmte Forschungsfragen, Themen und Felder. Eine erste Herausforderung besteht in der Einschätzung, in welchen Fällen ein sozialwissenschaftlicher Film vielversprechend, vorteilhaft und durchführbar erscheint. Filmische Darstellung bringt einige potenzielle Vorzüge mit sich, die ein schriftlicher Bericht nicht hat. Im Folgenden wird daher zunächst auf die Verbildlichung von Analysekategorien und danach auf den pragmatischeren Aspekt der Dissemination eingegangen.

Der Ausgangspunkt für alle Filme (egal welchen Genres) ist die Verwendung einer Kamera - eines Werkzeugs, das offensichtlich erforderlich ist, um die Bilder des Films herzustellen. Die Kamera ist ein zentrales (methodologisches) Instrument, das aus akademischer Sicht wichtige erkenntnistheoretische Fragen berührt. Man könnte lapidar formulieren, dass alle Sozialwissenschaftler/innen ihre Motive durch eine bestimmte »Linse « betrachten, aber für sozialwissenschaftliche Filmemacher/innen gilt dies buchstäblich und nicht nur metaphorisch. Der/Die Filmemacher/in sieht, präsentiert und bearbeitet durch die Kameralinse. In der Tat beginnt dieser Akt des Produzierens mit den Entscheidungen über die Kameraeinstellungen.

Eine ähnliche Überlegung, die kürzlich für die Organisationsforschung diskutiert wurde, befasst sich mit der Wirkmächtigkeit von Kameraeinstellungen: »[They] influence on the ways in which organizational space becomes available for analysis in video research « (Mengis et al. 2018, 19). Jeder Blickwinkel, den die Filmemacher/innen wählen, offenbart etwas von ihren selbstverständlichen Werten und bietet eine besondere Perspektive auf das Feld. Dieser Aspekt geht jedoch über die Frage der Perspektive hinaus. Die Kamera produziert verschiedene Forschungsschwerpunkte:

»Taken together, we can show that the combinations of camera angle and movement used to collect data (which we call video recording apparatuses) constitute a configuring device, which has a performative effect on the phenomenon of interest and does not simply record it $\ll$ (ebd., 306). 
Das gilt auch für den Abschlussfilm: Die Kamera ist in diesem Sinne auch Teil einer bestimmten Geschichte, die aus einer Vielzahl möglicher Geschichten ausgewählt wurde. Mengis et al. (2018) weisen auf die Konsequenzen dieser Diskussion hin: Unterschiedliche Kamerawinkel erzeugen unterschiedliche Räume, die beobachtbar werden.

Auch Sprache beziehungsweise geschriebener Text ist immer ein vermittelndes Medium. Stellt man Analyseergebnisse sprachlich dar, verbinden die Leser/innen damit bestimmte Vorstellungen und Bilder. Beschreibt man beispielsweise, wie eine der Studierendengruppen aus unserem Seminar es gemacht hat, das »Aufreißverhalten « in Clubs, so entstehen bei der Beschreibung der soziologischen Kategorien spezifische Bilder. Es stellt sich die Frage, ob es nicht auch für die soziologische Community spannend wäre, in einer audiovisuell geprägten Zeit audiovisuelle Darstellungsmittel zu wählen und damit die eigenen Vorstellungen der Kategorien durch sichtbare (ich sage absichtlich nicht reale) Bilder zu ersetzen. Mit allen Nachteilen, die sich ergeben, da natürlich auch die Wahl der Kameraposition und der Schnitt Positionierungen sind und die Konstruktion einer bestimmten Version von Wirklichkeit darstellen. Denn Fotografien und Filme sind »keine Spiegelbilder der Wirklichkeit, sondern nur Darstellungsformen [...], die ohne Analyse blind bleiben « (Flick 1995, 138). So wird gerade in der performativen Sozialwissenschaft darauf hingewiesen, dass durch die Reduktion auf Sprache viele Sinne vernachlässigt werden und eine vermeintliche Sicherheit und Genauigkeit in der Darstellung von Ergebnissen unterstellt wird (Parker 2004). Gerade durch eine Wissenschaftssprache, die ein breites Publikum ausschließt, wird diese Fokussierung auf den logos noch verstärkt. Hassard et al. (2018, 1411) fordern daher $\gg$ a more nuanced sense of affect and embodiment in video-based research $\ll$.

Darüber hinaus ist in Zweifel zu ziehen, ob die Suche nach einem Bild tatsächlich subjektiver ist als die Suche nach dem richtigen Wort für eine Typik. Das Problem der situativen Auswahl aus einer schier unbegrenzten Menge von Wortzusammenstellungen hat die Hermeneutik eindrucksvoll dargestellt (vgl. u. a. Froschauer und Lueger 2003). Das gilt auch für das Zoomen der Kamera. Der Umgang mit der Feder, beziehungsweise heute wohl eher der Tastatur, und der Umgang mit der Kamera sind beides höchst subjektive Prozesse. Der Lösungsansatz ist für beide Bereiche gleich: die intersubjektiv nachvollziehbare Darstellung des Forschungsprozesses, um die wissenschaftliche Gemeinschaft an der Produktion teilhaben zu lassen.

Ein pragmatischerer Aspekt in der Argumentation für den sozialwissenschaftlichen Film ist ein auf die Wissenschaftsvermittlung bezogener: Dissemination Strategies ist ein Begriff, der sich in den letzten Jahren bei der Drittmittelförderung zu einer fast unumgänglichen Bedingung entwickelt hat, um Projekte bei Fördereinrichtungen $»$ durchzubringen «. Hierbei geht es darum, die sozialwissenschaftlichen Ergebnisse der Öffentlichkeit zugänglich zu machen (im deutschsprachigen Raum als »Third Missi- 
on « diskutiert; dazu Henke, Pasternack und Schmid 2017). In diesem Zusammenhang wird etwa immer wieder die Verbreitung sozialwissenschaftlichen Wissens durch audiovisuelle Medien vorgeschlagen.

Mit einem sozialwissenschaftlichen Film soll jedoch mehr entstehen als - provokant formuliert - eine Marketingstrategie: nämlich ein Konzept innovativer Wissenschaftsvermittlung über disziplinäre Grenzen hinweg. So kann ein Film etwa Sprachbarrieren überwinden: Film kann ein potenzielles Publikum ansprechen, das mit Texten - zumal mit solchen, die in einer komplexen wissenschaftlichen Sprache verfasst sind - nicht erreicht werden kann. Der sozialwissenschaftliche Film bietet also die Möglichkeit, interkulturelle Kommunikation zu fördern, weil er weniger auf Sprache angewiesen ist als ein schriftlicher Text. Dies ist vermutlich nicht zuletzt ein Grund dafür, dass immer öfter am Rande sozialwissenschaftlicher Kongresse auch Filmarbeiten gezeigt werden.

Es ist mir ein Anliegen, klarzustellen, dass ich nicht der Meinung bin, dass sozialwissenschaftliche Filme alle Fragestellungen und methodischen Zugänge erfassen und abdecken können. Ich argumentiere auch in keiner Weise gegen die Notwendigkeit von Texten. Wir brauchen sowohl Filme als auch schriftliches Material in den Sozialwissenschaften, da sich beide Formen auf je unterschiedliche Weise mit Datenmaterial, Theorie und Rezeption befassen. Film alleine ist nicht in der Lage, Sozialität in ihrer Gesamtheit zu erfassen oder zu analysieren. Außerdem weisen jüngere Debatten auf die Möglichkeiten unserer nichtvisuellen Sinne (z. B. Tasten, Schmecken, Riechen) hin, unser Verständnis des Sozialen zu bereichern (Pink 2015; Riach und Warren 2015). Trotzdem möchte ich in diesem Artikel auf den Augensinn fokussieren und auf das Potenzial des Visuellen hinweisen: Es erweitert die Palette der Werkzeuge, mit denen Sozialwissenschaftler/innen ihr Fach verstehen und repräsentieren können.

Ich teile auch die Bedenken, dass unsere soziale Welt bereits stark visuell vergesellschaftet ist (Kavanagh 2014) und dass das Soziale durch eine solche monopolhafte Fokussierung auf einen singulären Sinn (»reliance on a singular sense «; Monthoux 2014) - genauso wie durch die Fokussierung auf den logos - eingeschränkt wird. Die Dominanz des Visuellen mag für die Populärkultur und sogar für die gelebte Erfahrung des Alltags zutreffen, trotzdem (hier formuliert für die Organisationsforschung): $\gg$ [We need the] visual as a necessary counterweight to redress the privileging of language in organizational research « (Bell, Warren und Schroeder 2014, 2). Es geht mir also nicht um das passive Erleben des oder die aktive Teilnahme am Sehen, das die Fernsehoder Smartphone-Rezeption mit sich bringt, sondern um das Potenzial, mit dem der Filmprozess einen » whole different way of looking at things « ermöglicht (Figgis 2007, 3). Der Einsatz der Filmkamera kann Forscher/inne/n eine weitere Möglichkeit bieten, kritische und engagierte Kommentare als Teil einer umfassenderen Untersuchung dessen zu entlocken, was empirische $\gg$ Daten « (Mitchell 2011) ausmacht, wenn etwa Organisationswissenschaftler/innen organisatorische Aspekte erschauen und ersehen. 
Zusammenfassend lässt sich festhalten: Der soziologische Endbericht in Textform muss und kann wahrscheinlich in vielen Fällen nicht ersetzt werden. Auch wir sahen in unserem Seminar zusätzlich zur Abgabe eines Films eine begleitende kurze Seminararbeit der Studierenden vor, in der die Forschungsarbeit und die filmische Darstellung erläutert und reflektiert wurden. In diesem Sinn kann der Film auch als eine Ergänzung zum schriftlichen Endbericht konzipiert werden. Jedenfalls aber birgt ein filmischer Endbericht Potenziale in Bezug auf Ergebnisdarstellung und Wissenschaftsvermittlung, die in mancher Hinsicht über jene eines schriftlichen Berichts hinausgehen.

\section{Zwischen Ästhetik und Gütekriterien: Eine Gratwanderung}

Interessanterweise sind die meisten Gütekriterien in der qualitativen Sozialforschung auf den Analyseprozess beschränkt. Viel seltener wird die Frage »Wie kommt man zum endgültigen Endbericht? « beantwortet. Immer wieder erweist sich in der Lehre die folgende Frage als eine der wichtigsten: Wie komme ich von einer großen Anzahl von Codes zu einem klaren Endbericht, zu einer »Storyline«, wie es Strauss und Corbin (1996 [1990]) genannt haben. In der Methodenliteratur ist nach wie vor wenig beschrieben (Girgensohn und Sennewald 2012), wie die Verbindung einzelner Kategorien in einen klaren Fließtext übersetzt werden kann (über die Wichtigkeit der Etablierung von Schreibzentren siehe Knorr und Brinkschulte 2019). Es scheint vorausgesetzt zu werden, dass Sozialwissenschaftler/innen, die analysieren können, gleichzeitig auch sprachliche Kompetenzen, beziehungsweise Kompetenzen in der sprachlichen Übersetzung der Analyseergebnisse, mitbringen. Diese »Übersetzungskompetenzen « sind jedoch für die Erstellung eines Textes nicht weniger wichtig als für die Produktion eines Filmes - sie werden nur weniger explizit gemacht.

Grundsätzlich gelten bei der Herstellung eines sozialwissenschaftlichen Films, was den Forschungsprozess betrifft, dieselben Kriterien wie bei anderen Forschungsprojekten. Für den Endbericht in Form eines Films gilt das Kriterium der »intersubjektiven Nachvollziehbarkeit« genauso wie für einen schriftlichen Endbericht. Aus meiner Sicht müssen ästhetische Kriterien (etwa die Bildqualität), im Vergleich zu Gütekriterien der sozialwissenschaftlichen Forschung, sekundäre Kriterien bleiben. Gleichzeitig hat performative Sozialwissenschaft auch den Anspruch, zu irritieren, subversiv zu sein und somit neue Perspektiven zu eröffnen. Genau deshalb bleibt es ein Problem, dass akademische Filme oft den Eindruck erwecken, nicht gestaltet worden und damit »langweilig « zu sein. Der Grund dafür ist, dass viele sozialwissenschaftliche Filme nicht der üblichen »Media Literacy« (Mikunda 2002) entsprechen, weil sie nicht die herkömmlichen, von uns leicht decodierbaren Gestaltungsmittel verwenden, mit de- 
nen wir in einer alltäglichen Fernseh- und Kinorezeption sozialisiert wurden - und die, positiv gewendet, eben andere Sinne ansprechen, als es die auf den Logos zentrierte Wissenschaft für gewöhnlich tut.

Davon abgesehen handelt es sich dabei aber auch um eine Grundsatzfrage im Kontext der Ergebnisdarstellung. Eine Frage, die sich allerdings auch beim Verfassen schriftlicher Texte stellt: nämlich inwieweit ich spannende, anschauliche sprachliche Mittel verwende, um mein Publikum zu erreichen; oder inwieweit ich möglichst wissenschaftlich-distanziert bleibe, um einer bestimmten Vorstellung von Wissenschaftlichkeit zu genügen. Provokant formuliert könnte man sich mancher Kritik an soziologischem Text anschließen: dass man sich hinter einer komplexen, hochformalisierten Sprache auch gut sprachlich, inhaltlich und in der eigenen Position zurückhaltend verstecken kann.

Bei der Gestaltung eines sozialwissenschaftlichen Films ergeben sich indes Probleme, die sich beim klassischen Schnitt für die Kino- und Fernsehproduktion nicht stellen. Wissenschaft hat, zumindest in vielen Bereichen und in unterschiedlichen Bedeutungen, einen gewissen Objektivitätsanspruch. Schnitt als Kulturtechnik ist aber als Teil einer Kunstform entstanden und die subjektive Position ist unumgänglich (und dem Kunstfeld immanent). Das ist natürlich auch beim Schreiben im akademischen Feld (bei qualitativer Forschung außerdem im Anspruch der intersubjektiven Nachvollziehbarkeit bereits wissenschaftlich gefasst) der Fall, doch muss hier die eigene Subjektivität stark reflektiert werden. Sozialwissenschaftlicher Film muss also einen schwierigen Spagat schaffen: zwischen dem wissenschaftlichen Anspruch, dem er verpflichtet ist, und der subjektiven Position, die mit der Gestaltung einhergeht.

Dazu einige Beispiele: Tonschnitt ist fast unumgänglich. Die Protagonist/inn/en sprechen oft sehr lange, doch muss man manchmal bestimmte Aussagen auf den Punkt bringen. Solche Fokussierungen helfen auch bei der Schärfung von Kategorien: Ton kann vom Interviewbild getrennt werden, das heißt, über den Ton des einen Bildes können gänzlich andere Bilder gelegt werden. Die Verwendung von Musik ist ein drittes Gestaltungsmittel. Musik emotionalisiert und steht der häufig geforderten Rationalität von Wissenschaft entgegen. Die Frage ist hier, ob Rationalität ein unumstößlicher Wert in den Wissenschaften ist (siehe dazu die Debatte um die Sinneslosigkeit in den Sozialwissenschaften in Abschnitt 2.2.). Auch dies ist aber kein Diskurs, der sich rein auf die bildliche Vermittlung von soziologischem Wissen bezieht. Wie schon erwähnt, wird die rationale Sprachverwendung in den Wissenschaften von verschiedenen Seiten kritisiert. Ob Wissenschaft emotional sein darf, ist also eine Frage, die sich gleichermaßen auf Text wie auf Bild bezieht. In all diesen Punkten gibt es keine klaren Vorgaben, sondern es werden schlichtweg Entscheidungen getroffen, die Konsequenzen haben. Unumgänglich ist, dass man diese Entscheidungen reflektiert und ihre intersubjektive Nachvollziehbarkeit sichergestellt ist. 
Ich möchte das an dem Film Auf den Spuren von Martha Muchow des Psychologen Günter Mey und des Filmemachers Günter Wallbrecht (2016) weiter veranschaulichen. Die beiden haben das Werk der Kindheitsforscherin Martha Muchow filmisch in Szene gesetzt und dies explizit als performative Sozialwissenschaft markiert (Mey 2018). Der Film von Mey und Wallbrecht siedelt sich dabei in einem Untergenre des sozialwissenschaftlichen Films an, nämlich dem der »Auseinandersetzung mit dem Werk und der Person von Forschenden « (Mey 2018, 13; siehe für eine Zusammenfassung von Filmen über Wissenschafter/innen: Heinze 2017). Der Film ist nicht nur von Interviews getragen - sogenannten »Talking Heads « (Briggs 2007, 557) -, sondern wird vielfältig dramatisiert. Eine sozialwissenschaftliche Geschichte filmisch zu erzählen, bedeutet vornehmlich, filmsprachliche, dramaturgische Mittel zu finden, um die Geschichte zu visualisieren. Mey und Wallbrecht haben sich neben den Talking Heads und geschickt eingesetztem historischen Material auch für ein schauspielerisches Element entschieden. Damit gehen sie entschieden eigene Wege, ist doch die Arbeit mit Schauspieler/inne/ $\mathrm{n}$ in einem sozialwissenschaftlichen Film ein umstrittenes dramaturgisches Mittel (Heider 2006).

In Auf den Spuren von Martha Muchow wird sehr anschaulich gemacht, dass Interviews, historisches Material, Szenen mit Schauspieler/inne/n, Abfilmen von Artefakten und Ähnliches vielfältig eingesetzt und miteinander kombiniert werden können, etwa wenn ein Interview auf der Tonebene bereits zu hören ist, auf der Bildebene jedoch noch ein historisches Artefakt gezeigt wird. Jede dieser Entscheidungen bedeutet eine Dramatisierung, etwa dann, wenn das Artefakt endgültig ausgeblendet wird und die Aufmerksamkeit nun ganz dem Interview gilt. Diese Schnittentscheidungen verweben die gesammelten Materialien und lassen sie zu einer visuellen Geschichte werden (Miko-Schefzig 2019).

Zusammenfassend zeigt sich, dass sich die wissenschaftliche Arbeit, die mit der Erstellung eines filmischen Endberichts endet, für den Großteil des Forschungsprozesses nicht vom sonst üblichen Weg unterscheidet. Erst sehr spät kommt der Unterschied zum Tragen: Während die Inhalte und Ergebnisse strukturiert werden und der Bericht verfasst wird, erfolgt beim sozialwissenschaftlichen Film an dieser Stelle die Arbeit am Filmmaterial.

\section{Sozialwissenschaft oder Filmstudium: Film im Curriculum der Sozialwissenschaften?}

Die Sozialwissenschaften werden - pointiert formuliert - auch ohne die Etablierung von Film weiterbestehen. Die Institutionalisierung von filmischer Arbeit im sozialwissenschaftlichen Curriculum ist keine Frage der Notwendigkeit, sondern eine Frage des 
Wollens. Fest steht auch, dass das sozialwissenschaftliche Studium keine Filmakademie ersetzen soll, will und kann. Es stellt sich aber die Frage, inwieweit sich etwa in Form von Tutorien doch ein Basiswissen in Schnitt und Gestaltung vermitteln lässt. Output und Nutzen einer derartigen Maßnahme können erst nach ein paar Semestern Lehre eingeschätzt werden. Möglich ist, dass der sozialwissenschaftliche Film dadurch weiter etabliert wird. Die Frage, ob Sozialwissenschaftler/innen die Kamera selbst in die Hand nehmen beziehungsweise Schnitttechniken und filmsprachliche Stilmittel kennen und anwenden können sollten, wird breit und uneindeutig diskutiert. Ich persönlich bin der Meinung, dass es schwierig ist, Filme zu machen (oder auch nur zu denken), ohne zumindest Grundkenntnisse in diesen Bereichen zu haben. Man kann es aber auch so sehen: Indem Wissenschaftler/innen und Filmemacher/innen zusammenarbeiten, entsteht eine Forschungsdynamik, » in which filmmakers, social theorists, participants and viewers alike are brought together in the same analytical space « (Hassard et al. 2018, 1417).

Jedenfalls aber ist die Verwendung von Film in soziologischen Studienplänen abseits der inhaltlichen Fragen auch eine der Finanzierung. Denn in Lehrveranstaltungen mit Film zu arbeiten, verursacht Zusatzkosten. Man muss, wie in unserem Seminar, methodische und technische Aspekte gleichzeitig vermitteln, muss im Optimalfall Tutor/inn/en engagieren, jedenfalls aber erhöhten zeitlichen und organisatorischen Aufwand betreiben. Was sich jedoch in den letzten zehn Jahren stark verändert hat, ist die Frage des Zugangs zum Equipment. Jedes Smartphone hat sowohl eine Kamera als auch ein Schnittprogramm. Tatsächlich haben sich die technischen Voraussetzungen seit dem Start unserer Lehrveranstaltung im Jahr 2007 völlig verändert. Während wir damals die Studierenden noch mit Kameras und Schnittprogrammen versorgen mussten, werden wir im Jahr 2019 nur selten danach gefragt. Dazu kommt, dass die Qualität der Smartphonekameras (und deren Mikrofone) stetig besser wird.

Die Frage, ob sich sozialwissenschaftlicher Film etablieren soll und wird, ist nicht zuletzt eine des (finanziellen) Willens der Universitäten. Hierzu ist anzumerken, dass auch eine verstärkte Zusammenarbeit mit anderen Universitätsinstituten, die ebenfalls mit Film arbeiten (wie etwa die Kultur- und Sozialanthropologie), sinnvoll wäre - sowohl auf der Ebene der Finanzierung und Organisation als auch auf der des inhaltlichen Austausches. In jüngerer Zeit haben sich außerdem die Publikationsmöglichkeiten von sozialwissenschaftlichen Filmen verbessert. Das an Harvard angedockte Onlinemedium Sensate Journal ist ein Beispiel dafür. Universitäten reagieren auf das Erstarken von Film in den Sozialwissenschaften, wie etwa das The Sensory Ethnography Lab (SEL) der Harvard Universität, ein Zusammenschluss unterschiedlicher Institute, die Film als sozialwissenschaftliche Methode weiterentwickeln (https://sel.fas.harvard.edu/index. html), zeigt. 


\section{$6 \quad$ Fazit}

Welche Conclusio kann ich nun ziehen? Der sozialwissenschaftliche Film wird die schriftliche Darstellung analytischer Ergebnisse und theoretischer Überlegungen in vielen Fällen nicht ersetzen können. Er eröffnet aber Möglichkeiten, die sprachliche Darstellungen nicht bieten. Allen voran die Unmittelbarkeit und Bildlichkeit der Erfahrung, zudem die Potenziale in Hinblick auf Wissenschaftskommunikation und -vermittlung. Ich denke, dass die audiovisuelle Darstellung in einer visuell geprägten Welt einen Mehrwert in der Erkenntnisvermittlung haben kann. Insbesondere eine Kombination von schriftlicher und filmischer Darstellung erscheint gewinnbringend. Von grundlegender Bedeutung ist in jedem Fall, dass die Gütekriterien qualitativer Sozialforschung, ebenso wie in anderen Forschungsprojekten, eingehalten werden und auch im Film intersubjektive Nachvollziehbarkeit gewährleistet ist. Zudem müssen Entscheidungen, die im Zuge der Filmgestaltung getroffen werden, sorgfältig reflektiert und begründet werden.

Um den sozialwissenschaftlichen Film verstärkt in die Lehre einzubinden, bräuchte es indes eine Grundsatzentscheidung - verbunden mit den entsprechenden finanziellen, organisatorischen und curricularen Strukturen. Damit aber wäre die Mitgestaltung eines Feldes ermöglicht, das sich nach wie vor in Entwicklung befindet. Die Sozialwissenschaften könnten sich aktiv an der Reflexion der angeführten Fragen und der Lösung der angesprochenen Probleme beteiligen und sich dadurch in diesem Bereich positionieren. Neue Erkenntnisse zur Ergebnisdarstellung, erweiterte Möglichkeiten der Wissenschaftsvermittlung und eine zusätzliche Dimension der Vielfalt innerhalb der Disziplin könnten das Resultat sein.

\section{Anmerkungen}

1 Die Basis dieses Artikels geht zurück auf einen früheren Text (Miko und Sardadvar 2008), der völlig überarbeitet wurde, unter anderem unter Rückgriff auf zwei neuere Publikationen (Miko-Schefzig 2019; Miko, Learmonth und McMurray 2019).

2 Für die ganze Szene siehe: https://www.youtube.com/watch?v=b_9-yVjtYXs\&feature=youtu. be (zugegriffen am 20.12.2019).

3 Siehe für Österreich etwa: https://www.oesterreich.gv.at/themen/bildung_und_neue_me dien/internet_und_handy__sicher_durch_die_digitale_welt/7/Seite.1720440.html und der neuen europäischen Datenschutzgrundverordnung (https://www.usp.gv.at/Portal.Node/usp /public/content/it_und_geistiges_eigentum/datenschutz_neu/rechte_von_betroffenen/31 3022.html (zugegriffen am 20.12.2019).

4 In Österreich ist das Einsetzen von Ethikkommissionen an Universitäten keine einheitliche Praxis. Im Gegenteil: An wenigen Universitäten sind solche Boards fix institutionalisiert.

5 Der Begriff »Codec« ist eine Paarung aus »Coder« (verschlüsseln) und »Decoder« (empfangen, verarbeiten). Als »Codec « wird in der Regel eine Kombination aus Kompressionsalgorithmus, Signalprotokollen und Dateiformaten bezeichnet. H.264 (auch bekannt als MPEG-4/AVC) ist ein bestimmter Standard zur Videokompression. 


\section{Literatur}

Banks, Marcus und David Zeitlyn. 2015. Visual methods in social research. London: Sage.

Bateson, Gregory und Margaret Mead. 1942. Balinese character: a photographic analysis. New York: New York Academy of Science.

Bell, Emma, Samantha Warren und Jonathan Schroeder. 2014. »The visual organization«. In The Routledge companion to visual organization, hrsg. v. Emma Bell, Samantha Warren und Jonathan Schroeder, 1-16. Abingdon: Routledge.

Berg, Eberhard und Martin Fuchs. 1993. Kultur, Soziale Praxis, Text. Die Krise der Ethnographischen Repräsentation. Frankfurt a. M.: Suhrkamp.

Briggs, Charles L. 2007. »Anthropology, interviewing, and communicability in contemporary society«. Current Anthropology 48 (4): 551-80.

Christianson, Marlys K. 2016. »Mapping the terrain: The use of video-based research in top-tier organizational journals«. Organizational research methods 21 (2): 1-27.

Denzin, Norman K. 1989. The research act. A theoretical introduction to sociological methods. New Jersey: Prentice-Hall.

Denzin, Norman K. 2000. »Reading Film - Filme und Videos als sozialwissenschaftliches Erfahrungsmaterial«. In Qualitative Forschung - ein Handbuch, hrsg. v. Uwe Flick, Ernst von Kardorff und Ines Steinke, 416-28. Reinbek bei Hamburg: Rowohlt.

Denzin, Norman K. 2001. »The reflexive interview and a performative social science«. Qualitative research 1 (1): 23-46.

Figgis, Mike. 2007. Digital film-making. London: Faber \& Faber.

Flaherty, Robert J. 1922. Nanook of the north. A story of life and love in the actual arctic. [Film.] USA: Robert J. Flaherty. Zugegriffen am 17.03.2019. https://www.imdb.com/title/tt0013427/.

Flick, Uwe. 2002. Qualitative Sozialforschung. Eine Einführung. Reinbek bei Hamburg: Rowohlt.

Froschauer, Ulrike und Manfred Lueger. 2003. Das qualitative Interview. Wien: UTB.

Girgensohn, Katrin und Nadja Sennewald. 2012. Schreiben lehren, Schreiben lernen: Eine Einführung. Darmstadt: Wissenschaftliche Buchgesellschaft.

Glaser, Barney G. und Anselm L. Strauss. 1967. The discovery of grounded theory: strategies for qualitative research. Chicago: Aldine.

Glass, Nel. 2008. »Interrogating the conventional boundaries of research methods in social sciences: the role of visual representation in ethnography". Forum Qualitative Sozialforschung/Forum: qualitative social research 9 (2): Art. 50. http://dx.doi.org/10.17169/fqs-9.2.391.

Glisovic, Smiljana, Leo Berkeley und Craig Batty. 2016. »The problem of peer review in screen production: exploring issues and proposing solutions«. Studies in Australasian Cinema 10 (1): 5-19.

Goffman, Erving. 1974. Frame analysis: an essay on the organization of experience. Cambridge: Harvard University Press.

Guiney Yallop, John J., Irene Lopez de Vallejo und Peter R. Wright. 2008. »Editorial: Overview of the performative social science special issue«. Forum Qualitative Sozialforschung/Forum: Qualitative Social Research 9 (2): Art. 64. http://dx.doi.org/10.17169/fqs-9.2.375.

Hassard, John, Diane Burns, Paula Hyde und John-Paul Burns. 2018. A visual turn for organizational ethnography: Embodying the subject in video-based research. Organization Studies 39 (10): 1403-1424.

Heider, Karl G. 2006. Ethnographic film. Austin, TX: University of Texas Press.

Heinze, Carsten. 2017. »Rezension: Auf den Spuren von Martha Muchow: ein Film von Günter Mey und Günter Wallbrecht«. Zeitschrift für Qualitative Forschung 18 (1): 159-65. https://doi.org/ 10.3224/zqf.v18i1.13. 
Heinze, Carsten und Thomas Weber. 2017. Medienkulturen des Dokumentarischen. Wiesbaden: Springer VS.

Henke, Justus, Peer Pasternack und Sarah Schmid. 2017. Mission, Die dritte: Die Vielfalt jenseits hochschulischer Forschung und Lehre: Konzept und Kommunikation der Third Mission. Berlin: Berliner Wissenschafts-Verlag.

Hohenberger, Eva. 1988. Die Wirklichkeit des Films. Dokumentarfilm - Ethnographischer Film - Jean Rouch. Hildesheim,Zürich \& New York: Olms.

Holm, Gunilla. 2008. »Photography as a performance«. Forum Qualitative Sozialforschung/Forum: Qualitative Social Research 9 (2): Art. 38, http://dx.doi.org/10.17169/fqs-9.2.394.

Jarzabkowski, Paula, Rebecca Bednarek und Laure Cabantous. 2015. „Conducting global teambased ethnography: methodological challenges and practical methods«. Human relations 68 (1): 3-33.

Kaczmarek, Jerzy. 2008. „Soziologischer Film - theoretische und praktische Aspekte«. Forum Qualitative Sozialforschung/Forum: Qualitative Social Research 9 (3), Art. 34. http://dx.doi.org/10. 17169/fqs-9.3.1164.

Kavanagh, Donncha. 2014. »The limits of ocularcentrism and organization«. In The Routledge companion to visual organization, hrsg. v. Emma Bell, Samantha Warren und Jonathan Schroeder, 416-28. Abingdon: Routledge.

Knoblauch, Hubert, Alejandro Baer, Eric Laurier, Sabine Petschke und Bernt Schnettler. 2008. »Visual Analysis. New Developments in the Interpretative Analysis of Video and Photography". Forum Qualitative Sozialforschung/Forum: Qualitative Social Research 9 (3), Art. 14. http://dx. doi.org/10.17169/fqs-9.3.1170.

Knorr, Dagmar und Melanie Brinkschulte. 2019. »Akademisches Schreiben lehren und lernen - Spektren einer prozessorientierten Schreibdidaktik«. Journal für Psychologie 27 (1): 51-71. https:// doi.org/10.30820/0942-2285-2019-1-51.

Kurt, Ronald. 2010. „Diener zweier Damen. Videobasierte Sozialforschung zwischen Datendokumentation und Filmproduktion«. In Videographie praktizieren, hrsg. v. Michael Corsten, Melanie Krug und Christine Moritz, 195-208. Wiesbaden: Springer.

Linstead, Stephen A. 2018a. »Feeling the reel of the real: Framing the play of critically affective organizational research between art and the everyday«. Organization Studies 39 (2-3): 319-44.

Linstead, Stephen A. 2018b. Black snow: The past lives on. [Film.] Großbritannien: All Rites Reversed \& Bellebete Productions. Zugegriffen am 17.09.2019. https://www.imdb.com/title/ tt7557886/.

Mey, Günter. 2018. »Performative Sozialwissenschaft und psychologische Forschung«. In Handbuch qualitative Forschung in der Psychologie, 2., akt. u. erw. Aufl., hrsg. v. Günter Mey und Katja Mruck, 1-25. Wiesbaden: Springer Reference Psychologie. https://doi.org/10.1007/978 -3-658-18387-5_29-1.

Mey, Günter und Günter Wallbrecht. 2016. Auf den Spuren von Martha Muchow. Lengerich: Pabst [DVD, 45 Min., engl. Untertitel, 33 Min. Bonusmaterial].

Mengis, Jeanne, Davide Nicolini und Mara Gorli. 2018. The video production of space: How different recording practices matter. Organizational research methods 21 (2): 288-315.

Miko, Katharina. 2013. „Visuelles `Nosing Aroundk. Zur theoretischen Fundierung visualisierter Wissenschaftskommunikation«. Soziale Welt 64 (1-2): 153-70.

Miko-Schefzig, Katharina. 2019. »Review Essay: Die sozialwissenschaftlich-filmische Ästhetisierung einer Pionierin der Kindheitsforschung: `Auf den Spuren von Martha Muchow«. Forum Qualitative Sozialforschung/Forum: Qualitative Social Research 20 (3): Art. 30. http://dx.doi.org/10. 17169/fqs-20.3.3394. 
Miko, Katharina und Raffael Frick. 2012. Warme Gefühle - vier Liebesgeschichten aus Österreich. [Film.] Wien: Nikolaus Geyrhalter Filmproduktion. Zugegriffen am 17.09.2019. https://www. filmportal.de/film/das-kind-und-die-welt_77054e85d15742c58feb898ce604d057.

Miko-Schefzig, Katharina, Mark Learmonth und Robert McMurray. 2020. »A different way of looking at things: the role of social science film in organization studies«. Organization [in Begutachtung].

Miko, Katharina und Karin Sardadvar. 2008. »Der ethnographische Film in der Soziologie: Möglichkeiten und Grenzen«. Onlinepublikation der Visuellen Soziologie, Universität Wien. Zugegriffen am 11.09.2019. http://www.univie.ac.at/visuellesoziologie/Publikation2008/ VisSozMikoSardadvar.pdf.

Miko, Katharina und Karin Sardadvar. 2010. »Das Unbehagen visuellen Wissens. Zur theoretischen Fundierung der Beziehung zwischen Geschlechterwissen und visuellem Wissen am Beispiel von Familienbildern«. In Körper - Wissen - Geschlecht. Geschlechterwissen \& soziale Praxis II, hrsg. v. Angelika Wetterer, 202-20. Sulzbach: Helmer.

Mikunda, Christian. 2002. Kino spüren. Strategien der emotionalen Filmgestaltung. Wien: facultas.

Mitchell, Claudia. 2011. Doing Visual Research. London: Sage.

Mohn, Elisabeth. 2002. Filming Culture. Spielarten des Dokumentierens nach der Repräsentationskrise. Oldenbourg: de Gruyter.

Mohn, Elisabeth. 2016. Kinder als Grenzgänger. Übergangspraktiken im Betreuungsalltag. [Film.] Berlin: dVb dohrmannVerlag.berlin. Zugegriffen am 17.09.2019. http://kamera-ethnographie. de/uploadfiles/documents/2010_120312_web_DVD_booklet_2016.pdf.

Monaco, James. 1997. Film verstehen. Reinbek bei Hamburg: Rowohlt.

Monthoux, Pierre Guillet. 2014. »Art, artist and aesthetics for organizational visual strategy«. In The Routledge companion to visual organization, hrsg. v. Emma Bell, Samantha Warren und Jonathan Schroeder, 146-61. Abingdon: Routledge.

Parker, Ian. 2004. »Criteria for qualitative research in psychology«. Qualitative Research in Psychology 1 (2): 95-106.

Pink, Sarah. 2007. Doing visual ethnography. Los Angeles, CA: Sage.

Pink, Sarah. 2015. Doing sensory ethnography. London: Sage.

Pfeifer, Jens. 2006. Dichte Teilnahme mit der Kamera. Der Film »Them and Me« von Stéphane Breton. Hamburg \&Münster: Lit-Verlag.

Riach, Kathleen und Samantha Warren. 2015. "Smell organization: Bodies and corporeal porosity in office work«. Human Relations 68 (5): 789-809.

Rose, Gillian. 2014. „On the relation between svisual research methods culture«. The Sociological Review 62 (1): 24-46.

Rouch, Jean und Edgar Morin. 1961. Chronique d'un été. [Film.] Zugegriffen am 17.09.2019. https:// www.imdb.com/title/tt0054745/.

Salovaara, Perttu. 2014. Leadership in spaces and places. [Film.] Finnland: Universität Tampere. Zugegriffen am 17.09.2019. https://vimeo.com/95709554.

Sauper, Hubert. 2005. Darwin's Nightmare. [Film.] Österreich: coop99.Zugegriffen am 17.09.2019. https://www.imdb.com/title/tt0424024/.

Schändlinger, Robert. 1998. Erfahrungsbilder: Visuelle Soziologie und dokumentarischer Film. Konstanz: UVK Medien.

Slutskaya, Natasha, Annilee M. Game und Ruth C. Simpson. 2018. »Better together: examining the role of collaborative ethnographic documentary in organizational research«. Organizational Research Methods 21 (2): 341-65.

Schnettler, Bernt und Frederik S. Pötzsch. 2007. „Visuelles Wissen«. In Handbuch Wissenssoziologie und Wissensforschung, hrsg. v. Rainer Schützeichel, 472-84. Konstanz: UVK. 
Soeffner, Hans-Georg und Jürgen Raab. 2004. „Sehtechniken. Die Medialisierung des Sehens: Schnitt und Montage als Ästhetisierungsmittel medialer Kommunikation«. In Auslegung des Alltags - der Alltag der Auslegung, hrsg. v. Hans-Georg Soeffner, 254-84. Konstanz: UTB.

Strauss, Anselm L. und Juliet M. Corbin. 1996 [1990]. Grounded Theory: Grundlagen qualitativer Sozialforschung. Weinheim: Psychologie Verlags Union.

Streeck, Jürgen. 2017. Self-making man. A day of action, life and language. New York: Cambridge University Press.

Taggart, Jonathan. 2016. Life off grid. [Film.] Kanada: Fighting Chance Films. Zugegriffen am 17.09.2019. https://www.imdb.com/title/tt4235130/.

Toraldo, Laura Marie, Gazi Islam und Gianluigi Mangia. 2018. Modes of knowing. Video research and the problem of elusive knowledges. Organizational Research Methods 21 (2), 438-465.

Vannini, Phillip. 2015. »Ethnographic film and video on hybrid television. Learning from the content, style, and distribution of popular ethnographic documentaries«. Journal of Contemporary Ethnography 44 (4): 391-416.

Weinberger, Eliot. 1994. »The camera people«. In Visualizing theory, hrsg. v. Lucien Taylor, 3-26. New York \& London: Routledge.

Wolff, Stephan. 1995. »Gregory Bateson \& Margaret Mead: `Balinese Character (1942) - Qualitative Forschung als disziplinierte Subjektivität«. In Handbuch qualitative Sozialforschung: Grundlagen, Konzepte, Methoden und Anwendungen, hrsg. v. Uwe Flick, Ernst von Kardorff, Heiner Keupp, Lutz von Rosenstiel und Stephan Wolff, 135-41. Weinheim: Beltz.

Wood, Martin und Sally Brown. 2011. "Lines of flight: Everyday resistance along England's backbone«. Organization 18 (4), 517-39.

Wood, Martin und Sally Brown. 2014. Lines of flight. [Film.] Großbritannien: University of York. Zugegriffen am 17.09.2019. https://www.imdb.com/title/tt1546411/.

Wood, Martin, Perttu Salovaara und Laurent Marti. 2018. »Manifesto for filmmaking as organisational research«. Organization 25 (6): 825-35.

\section{Die Autorin}

Katharina Miko-Schefzig, PD Dr.in phil., leitet das Kompetenzzentrum für empirische Forschungsmethoden der Wirtschaftsuniversität Wien. Ihre Arbeitsschwerpunkte sind qualitative Forschungsmethoden, interpretative Theorie, Sicherheits- und Polizeiforschung, visuelle Soziologie und sozialwissenschaftlicher Film.

Kontakt: PD Dr. ${ }^{\text {in }}$ Katharina Miko-Schefzig, Kompetenzzentrum für empirische Forschungsmethoden, Wirtschaftsuniversität Wien, Welthandelsplatz 1, A-1020 Wien; E-Mail: kmiko@wu.ac.at 